\title{
"It's a sign of weakness:" Masculinity, Help-Seeking Behaviors, and Gender-Specific Experiences Accessing VA PTSD Care
}

\section{Molly Silvestrini ( $D$ Mollidee.Silvestrini@va.gov )}

VA Puget Sound HCS Seattle Division: VA Puget Sound Health Care System https://orcid.org/00000001-9233-1277

Jessica A. Chen

VA Puget Sound HCS Seattle Division: VA Puget Sound Health Care System

\section{Research}

Keywords: Masculinity, PTSD, Gender, Veterans, Mental Health

Posted Date: November 23rd, 2021

DOI: https://doi.org/10.21203/rs.3.rs-1092195/v1

License: (c) (i) This work is licensed under a Creative Commons Attribution 4.0 International License.

Read Full License 


\section{Abstract}

Background: Male veterans delay post-traumatic stress disorder (PTSD) treatment and are less likely to engage in help-seeking behaviors or receive adequate mental health treatment. Male veterans face additional stigma seeking mental health care due to traditional masculine ideologies perpetuated by military culture. This study presents the gender-specific perspectives of veterans accessing VA PTSD care, focusing particularly on the help-seeking behaviors and barriers to care experienced by male veterans.

Methods: Semi-structured interviews were conducted with 25 U.S. veterans seeking treatment in VA primary care. Qualitative data analysis was coded using Atlas.ti, and thematic analysis was used to develop and refine themes. This study is part of a larger study examining veterans' initiation of PTSD treatment.

Results: Findings indicate that male veterans may be reluctant to initiate PTSD care due to stigma, distrust of the military or mental health care, and a desire to avoid reliving their trauma. Social support may encourage help-seeking behaviors among this population. Both male and female veterans reported a need for non-combat PTSD care and resources for military sexual trauma (MST).

Conclusions: Findings indicate that male veterans face unique challenges accessing mental health services and PTSD treatment, while both male and female veterans may benefit from increased VA services focused on MST and non-combat specific PTSD.

\section{Introduction}

Post-traumatic stress disorder (PTSD) is a mental disorder that is associated with significant health problems, such as higher rates of morbidity and physical impairment $(1,2)$, diminished quality of life (3), and increased mental health comorbidities, including depression (4). PTSD can also disrupt interpersonal relationships and family satisfaction (5), which may exacerbate mental health symptoms, as social support is associated with better psychological well-being and lower healthcare utilization (6). Among the U.S. military veteran population, PTSD is one of the most prevalent mental disorders (7), with studies estimating PTSD rates ranging from approximately 8 to $22 \%(8,9)$.

Previous research has identified significant gender differences between male and female veterans with PTSD and their experiences accessing VA care. Female veterans with PTSD have higher mental health, primary care, and emergency care use (10) and experience additional risk factors for PTSD, such as childhood abuse and interpersonal violence (11). Conversely, male veterans delay treatment between the onset of PTSD and help seeking (11) and are less likely to receive adequate mental health treatment (12). Men may also face additional stigma seeking mental health treatment due to traditional masculine ideologies (13), which can be perpetuated by military culture (14). Traditional constructions of hegemonic masculinity in the military perpetuate traits such as risk-taking, physical toughness, aggression, violence, emotional control, and overt heterosexual desire (15). Cultural expectations of femininity and masculinity significantly shape patient narratives when seeking care (16), and the complex "hypermasculinity" 
associated with war and militarism can make transition to civilian life challenging for veterans (17). In addition, masculine ideologies are associated with increased rates of suicide among military service members (18) and may affect male veterans' experiences and conceptualizations of trauma (19).

While quantitative research examining statistical associations between gender and health care utilization or rates of PTSD has been established $(10,20)$, as well as qualitative studies presenting the experiences of female veterans' accessing mental health care within the VA $(21,22)$, less research has focused on qualitative narratives of male veterans discussing barriers to PTSD treatment and help-seeking behaviors. Although there exists qualitative research examining barriers to PTSD care among the veteran population $(23,24)$, these studies have not focused on gender-specific barriers. This paper presents the perspectives of male and female veterans regarding VA PTSD care and explores the gender differences in their experiences accessing mental health services, focusing particularly on the barriers to care faced by male veterans with PTSD.

\section{Methods}

\section{Participants and Recruitment}

The data presented in this paper is part of a larger qualitative study, in which we conducted interviews with 25 veterans examining the initiation of PTSD services at the VA in the primary care setting. Participants were recruited from 9 primary care clinics with integrated behavioral health services; all clinics were part of one large VA healthcare system in the Northwest. Using the electronic health record, study staff identified 107 eligible patients who had an initial mental health appointment associated with a PTSD diagnosis ("index visit") between 04/01/2020 and 01/15/2021. As we sought to speak with patients who were initiating a new episode of PTSD care, patients were eligible only if they did not have any VA mental health visits in the two years prior. Eligible veterans were mailed a study information sheet and recruitment letter with an option to opt-out of the study; 8 participants opted out. Study staff called participants up to three times to invite them to participate in an interview; recruitment continued until thematic saturation was achieved. This study was approved by the local institutional review board.

\section{Data Collection}

Three members of the study evaluation team (a clinical psychologist, health science research specialist, and health systems engineer) developed a semi-structured interview guide designed to assess veterans' experiences discussing PTSD and mental health treatments with their providers. Between July 2020 and January 2021, two members of the study team trained in qualitative research and one trained in usability testing conducted semi-structured interviews with 25 veterans who had a recent, initial appointment for PTSD with a mental health provider in VA primary care. Before each interview, the interviewer verbalized the information statement detailing all aspects of study involvement with the participant, then obtained informed consent to be interviewed and recorded. Interviews were recorded and professionally transcribed. Each interview lasted approximately 60-90 minutes. After successful completion of the postinterview surveys, participants were compensated $\$ 50$ via check for their participation. 


\section{Data Analysis}

Atlas.ti (Version 8, Berlin, Scientific Software Development) was used to manage codes, organize data, and develop concepts. Deductive codes derived from questions in the interview guide were used to form an initial codebook and establish general domains for analysis. Two members of the study team independently reviewed and line-by-line coded two transcripts, then met to discuss the codebook in order to add inductive codes that would further categorize veterans' experiences with PTSD treatment in VA primary care. The two analysts coded the remaining transcripts independently to establish consensus. Qualitative data were then analyzed using thematic analysis, a method for identifying, analyzing, organizing, describing, and reporting themes found within a data set (25). Thematic analysis is useful in examining the perspectives of research participants, generating unanticipated insights, and highlighting similarities and differences in a large data set $(25,26)$. Using this method, the analysis team closely examined code reports generated by Atlas.ti in order to establish trends in the data, which were then refined to identify salient themes. Themes developed through this process were then discussed by the research team to ensure they accurately reflected the depth and nuances of the data. In addition to broader themes regarding experiences accessing care and perspectives of various PTSD treatments, the team also identified several gender-specific themes related to male and female veterans' perspectives and experiences accessing PTSD treatment, particularly barriers to care faced by male veterans. These findings are presented and discussed in the following sections.

\section{Results}

Of the 25 veterans recruited in this sample, 14 were male (56\%) and 11 were female (44\%). 13 participants identified as White (52\%), 4 identified as Black (16\%), 2 as Asian (8\%), 2 as American Indian (8\%), and 3 as more than one race (12\%). 2 participants identified as Hispanic/Latinx (8\%). Participant ages ranged from 22 to 72 , with an average of approximately 45 years. For the index visit (PTSD appointment that determined eligibility), 13 (52\%) participants met with their provider via video, 8 (32\%) had a phone visit, and 4 (16\%) saw their provider in person.

\section{Reluctance of Male Veterans to Seek PTSD Mental Health Treatment}

Many male veterans reported a general reluctance to seek mental health treatments for PTSD. Several reported an unwillingness to seek treatment because of distrust with mental health care or the belief that PTSD was not a legitimate mental health disorder. One veteran said: "When I first started recognizing the symptoms, the military told me, you don't have PTSD, you just have post-war issues. And that completely turned me off to anything, I didn't have faith in them or the system. So it was, yeah, I was totally turned off to the system until it got so bad that I had no choice." Another veteran said, "It took my counselor a lot to get me to open up and gain my trust. I think that's important to know." A third veteran explained, "I come from a family of all boys. We've all served overseas active duty in different military branches. If you 
sat my brothers and I down in a room at any point before May, all of my brothers and I would say pfft, I don't have PTSD, that's for the guys that are trying to get claims or whatever. PTSD is an administrative thing, I don't have PTSD, and honestly, most people don't have it. So I was just very ignorant and naïve, and fearful of the whole topic."

Male veterans also reported unwillingness to seek care because they did not want to discuss their mental health struggles with others or perceived treatment as a sign of weakness. One veteran said, "So it's one of those things that, it's not a thing to be proud of, do you know what l'm saying? I'm old school, you don't talk about your feelings. You kind of try to bottle them up and take care of them, but you can't take care of them on your own anymore. So then you need help from somewhere, and you have to get to the point where you want the help and ask for it. No, it's a sign of weakness, I feel, it's a point of weakness." Another veteran said, "[Counseling] was a foreign idea to me. And it wasn't that I didn't think it would help me, that I didn't need help, it's just that it was intimidating to admit that you're not whole. That you're not functioning like you might be." A third veteran stated, "It's personal. Like I said, I do not discuss, and that's my biggest problem, that might be one of the reasons I do have PTSD, because I normally try to keep everything to myself." Another veteran explained why he was unwilling to seek treatment: "I've never talked to anybody about this before. So, it's a little different of a road for me to travel down, because I'm a protector, I'm not one to come back and complain about something that's happened, I just handle it on my own and be able to make sure that the people around me are protected or safe."

Several veterans reported attempting to avoid mental health services before finally seeking treatment. One veteran spoke about the first time he received PTSD care: "For me, it was pretty bad. So, to relieve it was a lot of anxiety. Trying to avoid it. You don't want to relieve it, you don't want to talk about it, you just, in my mind, you just want to put it behind you and leave it there." He went on to say, "Because some of us vets, whatever trauma you deal with in your life, we try to put that stuff at the back of our mind, we try to avoid it, but we don't realize that it's causing us more pain from trying to avoid it than from talking and trying to work through it." Another veteran described their experience receiving PTSD counseling: "I try to, what do you call it? Put it in the back of my head and not think about stuff, and it brought it to the front of my head, and I kept reliving it, got more tense, I isolated myself more and that kind of stuff." Another stated, "I think there's a lot of veterans out there who have PTSD who never seek help. I just stumbled into this. We need to find some way to make it more advertised out there."

\section{Social Support Encourages Male Veterans' Help-Seeking Behaviors}

Several veterans reported that their families or friends encouraged them to seek PTSD care. One veteran who had been experiencing anger management issues reported that his wife eventually convinced him to seek PTSD treatment: "She finally just said, look, you need to get help. And when women tell us men to get help, if we're smart, we listen." Another reported, "I wasn't really feeling like myself. I felt like I was kind of reclusive, so I was just talking to my family, and they were like, if you need to see somebody about PTSD because none of us know what you're going through, talk to the VA." One veteran spoke about the 
influence that his children and social support members had on his decision to seek care: "I retired in 2006 and l've been out here ever since then, on my own, figuring out how to take care of myself the best way I know how, and if it wasn't for my kids and always wanting to set a good example for my kids, and having my support group and people always depending on me, asking me for help, it takes my mind of stuff. At the same time, I know that I've got people I can go and do stuff with to help me better adjust." Another veteran said, "I don't know how many vets on their own went into treatment. One of my best friends took me by the hand and had preloaded the introduction with the counselor and introduced me to her, and then he walked out and left me there with her. Otherwise, I never would have went in."

\section{Need for Additional Services in VA PTSD Care}

Both male and female veterans identified a demand for more inclusive PTSD care, such as resources that address military sexual trauma (MST) and are not combat specific. One male veteran said, "It's a deterrent to a lot of people when combat is right there, the first thing. Because, maybe up until a year or two ago, if you asked me, I would've said, yeah, PTSD is for the combat people who shot someone or got shot at. So, it kind of frustrates me when I see the combat, combat, combat. Because the majority of the people in the military aren't combat, and don't shoot anybody, and have a lot more reasons to experience PTSD." One female veteran disclosed, "My PTSD comes from MST, so it's not like I went to war and got shot at or anything like that. It was something completely different." Several other veterans reported a need for MST-specific PTSD care. Another female veteran said, "You'll see a lot more tailored groups like veterans with PTSD who are typically through combat and things like that. But mine happened through MST, and it's almost like they broke it up. Like they broke up PTSD and MST, but it really needs to be one unit thing, like PTSD because of MST." A male veteran who sought PTSD treatment stated, "I think we need to help the soldiers who have sexual trauma. With more and more female veterans speaking out and talking about their experiences in combat, let alone their trials and tribulations through active-duty period, I think that should be addressed somewhere."

\section{Discussion}

This qualitative study presents the gender-specific experiences of veterans with PTSD accessing VA mental health services and is unique in describing the perspectives that may be specific to male veterans. This research identifies 3 major findings: 1) Male veterans reported a reluctance to seek PTSD mental health treatment; 2) Social support may encourage male veterans' help-seeking behavior; and 3) Both male and female veterans identified a need for additional inclusive services in VA PTSD care, particularly a need for non-combat specific PTSD care and resources that address MST.

Male veterans in this sample reported a reluctance or unwillingness to seek PTSD mental health care at the VA due to stigma, distrust of the military or mental health care, and a desire to avoid reliving their trauma. Male veterans believed that seeking care is "a sign of weakness" or that in pursuing treatment, a veteran would have to "admit that you're not whole." Male veterans also expressed a desire to avoid discussing their mental health struggles or emotions with others, stating a preference to "bottle them up" or "keep everything to myself." Additionally, male veterans reported wanting to suppress their trauma, and 
that they would prefer to "put it behind you and leave it there," "avoid it," or "put it in the back of my head" so they wouldn't have to "relive" any traumatic events. One veteran felt that he "didn't have faith" in the military mental health system because he was incorrectly told he did not have PTSD.

Previous research suggests that internalized stigma is associated with increased psychiatric symptoms, produces unconscious views against seeking care, and encourages avoidant behavior (27). Denial, stigma, and fear of harming military career have been found to contribute to avoidance of help-seeking behaviors among veterans, and higher perceived public stigma of treatment seeking is significantly associated with lower treatment utilization (28). In addition, veterans have reported a preference for handling their mental health problems on their own (29) and have concerns regarding healthcare privacy and distrust of the VA healthcare system (30). This research aligns with our findings regarding male veterans' reluctance to engage in help-seeking for PTSD treatment.

Male veterans may experience additional pressure to avoid help-seeking due to traditional masculinity norms perpetuated by society and the military (31), which can impede veterans from seeking mental health services due to a fear of being perceived as cowardly or weak (32). One study found that $65 \%$ of veterans surveyed after combat duty believed that seeking mental health services signified weakness and cowardice, and $60 \%$ believed that they would be treated differently by their leaders and peers if they disclosed any mental health issues (33). Previous research has established that rigid commitment to hegemonic masculine ideals and gender roles are associated with PTSD symptoms (34). A review of PTSD treatment engagement among veterans found that endorsement of a traditional masculinity ideology fosters a culture in which experiencing a traumatic event is emasculating, and that endorsing beliefs that conform to traditional masculinity - such as emotional stoicism and stigmatizing one's emotions - are associated with PTSD severity and difficulties with functioning (14). In another study, male veterans who endorsed higher levels of emotional toughness were more likely to screen positive for PTSD and depression (12). However, research also indicates that masculinity can be reconstructed as a resource for dealing with PTSD by developing positive masculine identities associated with mental health, being in control, and help-seeking (35). These studies illustrate the relationship between traditional masculinity and perceptions of mental health, which is integral to the discussion of gender-specific barriers faced by male veterans.

Our findings also indicate that social support involvement may facilitate help-seeking behaviors among male veterans. Male veterans recounted instances in which their family or friends encouraged them to seek treatment when they would not have done so otherwise; one veteran decided to seek mental health services in order to set an example for his children, while another veteran stated that if his friend had not gone with him to a counseling appointment, he "never would have went in." Previous research has identified social support as a predictor for mental health help-seeking behavior and treatment engagement among veterans (36), and found that veterans reporting greater satisfaction with their social networks were less likely to be at elevated risk of suicide compared to veterans with lower satisfaction (37). Although a strong social support network can be beneficial, veterans with PTSD report increased difficulties in their relationships with romantic partners, less cohesion in their families, less social support, 
poor social functioning, and lower life satisfaction (38). Poor perceived social support among veterans with PTSD is associated with increased aggression (39), more severe PTSD symptoms (40), as well as increased impairment and suicidal ideation (41). These studies support our findings and highlight the impact of social support on male veteran help-seeking behaviors.

Both male and female veterans identified a need for additional VA PTSD services, including resources that address non-combat specific PTSD and MST. Although presentation of PTSD may differ between combat and non-combat veterans (42), previous research suggests that non-combat veterans face similar trauma frequency and negative emotions as combat veterans (43), indicating that there is a need for PTSD care that acknowledges the mental health struggles of non-combat veterans. In addition, both male and female veterans with PTSD report higher rates of MST than the general population, and non-combat veterans report higher rates of MST than their combat counterparts (44). Significant gender differences exist among veterans with MST; female veterans are more likely to drop out of VA care (45), while male veterans are less likely to use VA MST-related care (46) and delayed MST treatment longer than women (47). Both male and female veterans who report experiencing MST have higher rates of PTSD than those who have experienced other forms of trauma (48). Although PTSD and MST are clearly linked, it is important that they not be used interchangeably in health care settings so that veteran mental health consequences can be correctly managed (49). In a qualitative exploration of MST survivors' concerns regarding VA care, participants described wanting additional VA support for MST, increased continuity of care, and access to a variety of treatment options, such as holistic or gender-specific services (50). To address this gap in care, future VA PTSD interventions should address MST and non-combat specific PTSD.

\section{Limitations And Future Directions}

The findings presented here are part of a larger qualitative study focused on examining the initiation of PTSD services at the VA. Therefore, questions regarding gender-specific barriers to care were not part of the formal interview guide and were not standardized across the entire study population. Themes discussed here were introduced organically by participants and identified by the study team during analysis. Though the topic of gender-specific experiences was not originally part of this larger study's research aim, we believe that the narratives presented here are salient, distinctive perspectives of veterans who expressed challenges accessing mental health care and suggestions for improvement. In addition, this study did not assess the experiences of veterans whose gender identity falls outside the gender binary, such as genderfluid or nonbinary. We hope that future research focuses on the gender-specific barriers to PTSD care in order to inform VA interventions and improve care for all veterans with PTSD.

\section{Conclusions}

This research presents the experiences and perspectives of veterans accessing VA PTSD care, with a particular focus on the gender-specific barriers faced by male veterans. Findings indicate that male veterans may be reluctant to initiate PTSD care due to stigma, distrust of the military or mental health 
care, and a desire to avoid reliving their trauma, and that social support may encourage help-seeking behaviors among this population. In addition, findings suggest that both male and female veterans may benefit from increased VA services focused on MST and non-combat specific PTSD.

\section{Declarations}

Ethics approval and consent to participate: Informed consent was obtained from all subjects. This study was reviewed and approved by the VA Puget Sound Institutional Review Board. This research was performed in accordance with the Declaration of Helsinki and all methods were carried out in accordance with VA guidelines and regulations.

Consent for publication: Not applicable.

Availability of data and materials: The datasets used and/or analyzed during the current study are available from the corresponding author on reasonable request.

Competing interests: The authors declare they have no competing interests. The views expressed in this article are those of the authors and do not necessarily reflect the position or policy of the Department of Veterans Affairs.

Funding: This material is based upon work supported by the Department of Veterans Affairs, Veterans Health Administration, Office of Research and Development, Health Services Research and Development (IK2HX002866).

Author's contributions: MS and JAC made substantial contributions to the conception and design of the work; the acquisition, analysis, and interpretation of data; and drafted the work and substantially reviewed it. All authors read and approved the final manuscript, and all authors agreed both to be personally accountable for the author's own contributions and to ensure that questions related to the accuracy or integrity of any part of the work, even ones in which the author was not personally involved, are appropriately investigated, resolved, and the resolution documented in the literature.

\section{References}

1. Pacella ML, Hruska B, Delahanty DL. The physical health consequences of PTSD and PTSD symptoms: a meta-analytic review. J Anxiety Disord. 2013 Jan;27(1):33-46.

2. Hall KS, Beckham JC, Bosworth HB, Sloane R, Pieper CF, Morey MC. PTSD is negatively associated with physical performance and physical function in older overweight military Veterans. J Rehabil Res Dev. 2014;51(2):285-95.

3. Pagotto LF, Mendlowicz MV, Coutinho ESF, Figueira I, Luz MP, Araujo AX, et al. The impact of posttraumatic symptoms and comorbid mental disorders on the health-related quality of life in treatment-seeking PTSD patients. Compr Psychiatry. 2015 Apr;58:68-73. 
4. Nichter B, Norman S, Haller M, Pietrzak RH. Psychological burden of PTSD, depression, and their comorbidity in the U.S. veteran population: Suicidality, functioning, and service utilization. J Affect Disord. 2019 Sep 1;256:633-40.

5. Vogt D, Smith BN, Fox AB, Amoroso T, Taverna E, Schnurr PP. Consequences of PTSD for the work and family quality of life of female and male U.S. Afghanistan and Iraq War veterans. Soc Psychiatry Psychiatr Epidemiol. 2017;52(3):341-52.

6. Adams RE, Urosevich TG, Hoffman SN, Kirchner HL, Hyacinthe JC, Figley CR, et al. Social Support, Help-Seeking, and Mental Health Outcomes Among Veterans in Non-VA Facilities: Results from the Veterans' Health Study. Mil Behav Health. 2017 May 19;5(4):393-405.

7. Williamson V, Stevelink SAM, Greenberg K, Greenberg N. Prevalence of Mental Health Disorders in Elderly U.S. Military Veterans: A Meta-Analysis and Systematic Review. Am J Geriatr Psychiatry. 2018 May;26(5):534-45.

8. Goldberg J, Magruder KM, Forsberg CW, Friedman MJ, Litz BT, Vaccarino V, et al. Prevalence of PostTraumatic Stress Disorder in Aging Vietnam-Era Veterans: Veterans Administration Cooperative Study 569: Course and Consequences of Post-Traumatic Stress Disorder in Vietnam-Era Veteran Twins. Am J Geriatr Psychiatry. 2016 Mar;24(3):181-91.

9. Wisco BE, Marx BP, Wolf EJ, Miller MW, Southwick SM, Pietrzak RH. Posttraumatic stress disorder in the US veteran population: results from the National Health and Resilience in Veterans Study. J Clin Psychiatry. 2014 Dec;75(12):1338-46.

10. Maguen S, Cohen B, Cohen G, Madden E, Bertenthal D, Seal K. Gender differences in health service utilization among Iraq and Afghanistan veterans with posttraumatic stress disorder. J Womens Health (Larchmt). 2012 Jun;21(6):666-73.

11. Lehavot K, Katon JG, Chen JA, Fortney JC, Simpson TL. Post-traumatic Stress Disorder by Gender and Veteran Status. Am J Prev Med. 2018 Jan;54(1):e1-9.

12. Hoerster KD, Malte CA, Imel ZE, Ahmad Z, Hunt SC, Jakupcak M. Association of perceived barriers with prospective use of VA mental health care among Iraq and Afghanistan veterans. Psychiatr Serv. 2012 Apr;63(4):380-2.

13. Kuehn BM. Men face barriers to mental health care. JAMA. 2006 Nov 15;296(19):2303.

14. Neilson EC, Singh RS, Harper KL, Teng EJ. Traditional masculinity ideology, posttraumatic stress disorder (PTSD) symptom severity, and treatment in service members and veterans: A systematic review. Psychology of Men \& Masculinities. 2020 Jan 27;

15. Hinojosa R. Doing hegemony: military, men, and constructing a hegemonic masculinity. J Mens Stud. 2010 Apr 1;18(2):179-94.

16. Ahlsen B, Bondevik H, Mengshoel AM, Solbrække KN. (Un)doing gender in a rehabilitation context: a narrative analysis of gender and self in stories of chronic muscle pain. Disabil Rehabil. 2014;36(5):359-66.

17. Bulmer S, Eichler M. Unmaking militarized masculinity: veterans and the project of military-to-civilian transition. Critical Military Studies. 2017 May 4;3(2):161-81. 
18. Braswell H, Kushner HI. Suicide, social integration, and masculinity in the U.S. military. Soc Sci Med. 2012 Feb;74(4):530-6.

19. Fox J, Pease B. Military deployment, masculinity and trauma: reviewing the connections. J Mens Stud. 2012 Jan 1;20(1):16-31.

20. Gaffey AE, Burg MM, Rosman L, Portnoy GA, Brandt CA, Cavanagh CE, et al. Baseline Characteristics from the Women Veterans Cohort Study: Gender Differences and Similarities in Health and Healthcare Utilization. J Womens Health (Larchmt). 2021 Jul;30(7):944-55.

21. Kehle-Forbes SM, Harwood EM, Spoont MR, Sayer NA, Gerould H, Murdoch M. Experiences with VHA care: a qualitative study of U.S. women veterans with self-reported trauma histories. BMC Womens Health. 2017 May 30;17(1):38.

22. Ingelse K, Messecar D. Rural women veterans' use and perception of mental health services. Arch Psychiatr Nurs. 2016 Apr;30(2):244-8.

23. Hundt NE, Helm A, Smith TL, Lamkin J, Cully JA, Stanley MA. Failure to engage: A qualitative study of veterans who decline evidence-based psychotherapies for PTSD. Psychol Serv. 2018 Nov;15(4):536-42.

24. Possemato K, Wray LO, Johnson E, Webster B, Beehler GP. Facilitators and barriers to seeking mental health care among primary care veterans with posttraumatic stress disorder. J Trauma Stress. 2018 Oct 19;31(5):742-52.

25. Braun V, Clarke V. Using thematic analysis in psychology. Qual Res Psychol. 2006 Jan;3(2):77-101.

26. King N. Using templates in the thematic analysis of text. Essential guide to qualitative methods in organizational research. 1 Oliver's Yard, 55 City Road, London EC1Y 1SP United Kingdom: SAGE Publications Ltd; 2004. p. 256-70.

27. Drapalski AL, Lucksted A, Perrin PB, Aakre JM, Brown CH, DeForge BR, et al. A model of internalized stigma and its effects on people with mental illness. Psychiatr Serv. 2013 Mar 1;64(3):264-9.

28. Kulesza M, Pedersen E, Corrigan P, Marshall G. Help-Seeking Stigma and Mental Health Treatment Seeking Among Young Adult Veterans. Mil Behav Health. 2015 Jun 26;3(4):230-9.

29. Hoge CW, Castro CA, Messer SC, McGurk D, Cotting DI, Koffman RL. Combat duty in Iraq and Afghanistan, mental health problems, and barriers to care. N Engl J Med. 2004 Jul 1;351(1):13-22.

30. Cheney AM, Koenig CJ, Miller CJ, Zamora K, Wright P, Stanley R, et al. Veteran-centered barriers to VA mental healthcare services use. BMC Health Serv Res. 2018 Jul 31;18(1):591.

31. Lorber W, Garcia HA. Not supposed to feel this: traditional masculinity in psychotherapy with male veterans returning from Afghanistan and Iraq. Psychotherapy (Chic). 2010 Sep;47(3):296-305.

32. Zinzow HM, Britt TW, McFadden AC, Burnette CM, Gillispie S. Connecting active duty and returning veterans to mental health treatment: interventions and treatment adaptations that may reduce barriers to care. Clin Psychol Rev. 2012 Dec;32(8):741-53.

33. Hoge CW, Terhakopian A, Castro CA, Messer SC, Engel CC. Association of posttraumatic stress disorder with somatic symptoms, health care visits, and absenteeism among Iraq war veterans. Am J 
Psychiatry. 2007 Jan;164(1):150-3.

34. Christiansen DM, Berke ET. Gender- and Sex-Based Contributors to Sex Differences in PTSD. Curr Psychiatry Rep. 2020 Mar 3;22(4):19.

35. Caddick N, Smith B, Phoenix C. Male combat veterans' narratives of PTSD, masculinity, and health. Sociol Health IIIn. 2015 Jan;37(1):97-111.

36. Porcari C, Koch El, Rauch SAM, Hoodin F, Ellison G, McSweeney L. Predictors of Help-Seeking Intentions in Operation Enduring Freedom and Operation Iraqi Freedom Veterans and Service Members. Mil Med. 2017 May;182(5):e1640-7.

37. Jakupcak M, Vannoy S, Imel Z, Cook JW, Fontana A, Rosenheck R, et al. Does PTSD moderate the relationship between social support and suicide risk in Iraq and Afghanistan War Veterans seeking mental health treatment? Depress Anxiety. 2010 Nov;27(11):1001-5.

38. Tsai J, Harpaz-Rotem I, Pietrzak RH, Southwick SM. The role of coping, resilience, and social support in mediating the relation between PTSD and social functioning in veterans returning from Iraq and Afghanistan. Psychiatry. 2012;75(2):135-49.

39. Schnurr PP, Lunney CA, Sengupta A. Risk factors for the development versus maintenance of posttraumatic stress disorder. J Trauma Stress. 2004 Apr;17(2):85-95.

40. Brewin CR, Andrews B, Valentine JD. Meta-analysis of risk factors for posttraumatic stress disorder in trauma-exposed adults. J Consult Clin Psychol. 2000 Oct;68(5):748-66.

41. Debeer BB, Kimbrel NA, Meyer EC, Gulliver SB, Morissette SB. Combined PTSD and depressive symptoms interact with post-deployment social support to predict suicidal ideation in Operation Enduring Freedom and Operation Iraqi Freedom veterans. Psychiatry Res. 2014 May 30;216(3):35762.

42. Adler AB, Castro CA. An occupational mental health model for the military. Mil Behav Health. 2013 Jan;1(1):41-5.

43. Macia KS, Raines AM, Maieritsch KP, Franklin CL. PTSD networks of veterans with combat versus non-combat types of index trauma. J Affect Disord. 2020 Dec 1;277:559-67.

44. Murdoch M, Polusny MA, Hodges J, O'Brien N. Prevalence of in-service and post-service sexual assault among combat and noncombat veterans applying for Department of Veterans Affairs posttraumatic stress disorder disability benefits. Mil Med. 2004 May;169(5):392-5.

45. Washington DL, Bean-Mayberry B, Riopelle D, Yano EM. Access to care for women veterans: delayed healthcare and unmet need. J Gen Intern Med. 2011 Nov;26 Suppl 2:655-61.

46. Turchik JA, Pavao J, Hyun J, Mark H, Kimerling R. Utilization and intensity of outpatient care related to military sexual trauma for veterans from Afghanistan and Iraq. J Behav Health Serv Res. 2012 Jul;39(3):220-33.

47. O'Brien C, Keith J, Shoemaker L. Don't tell: Military culture and male rape. Psychol Serv. 2015 Nov;12(4):357-65. 
48. Kang H, Dalager N, Mahan C, Ishii E. The role of sexual assault on the risk of PTSD among Gulf War veterans. Ann Epidemiol. 2005 Mar;15(3):191-5.

49. Williamson MLC, Holliday R, Holder N, North CS, Surís A. A reexamination of military sexual trauma and posttraumatic stress disorder. Psychiatr Ann. 2017 Mar 1;47(3):134-8.

50. Monteith LL, Bahraini NH, Gerber HR, Dorsey Holliman B, Schneider AL, Holliday R, et al. Military sexual trauma survivors' perceptions of veterans health administration care: A qualitative examination. Psychol Serv. 2020 May;17(2):178-86.

\section{Tables}

Table 1. Demographic Information

\begin{tabular}{ll} 
Total N & 25 \\
\hline Age M (SD), years & $45(13)$ \\
\hline Gender & \\
\hline Female & $11(44 \%)$ \\
\hline Male & $14(56 \%)$ \\
\hline Race & \\
\hline American Indian or Alaska Native & $2(8 \%)$ \\
\hline Asian & $2(8 \%)$ \\
\hline Black or African American & $4(16 \%)$ \\
\hline White & $13(52 \%)$ \\
\hline More than one & $3(12 \%)$ \\
\hline Unknown & $1(4 \%)$
\end{tabular}

Ethnicity

\begin{tabular}{ll}
\hline Hispanic or Latinx & $2(8 \%)$ \\
\hline Not Hispanic or Latinx & $22(88 \%)$ \\
\hline Unknown & $1(4 \%)$ \\
\hline
\end{tabular}

Type of Visit

\begin{tabular}{ll}
\hline Video & $13(52 \%)$ \\
\hline Phone & $8(32 \%)$ \\
\hline In-Person & $4(16 \%)$
\end{tabular}

